\title{
Plastic Surgery Innovation Through War, Disaster and Pandemic
}

\author{
Kayla Clodfelter ${ }^{1}$, Brian Mailey ${ }^{2}$, Aladdin H. Hassanein ${ }^{1}$ \\ ${ }^{1}$ Indiana University School of Medicine, ${ }^{2}$ Southern Illinois University School of Medicine
}

Introduction and Objectives: Mass casualties from modern warfare in World War I and advancements in medicine resulted in survivors with deformities. The field of Plastic Surgery developed from a need to reconstruct soft tissue injuries in these soldiers. Wars and disasters provide unique situations which push the development of new techniques that impact the field. The objective of this work was to identify innovations in plastic surgery that emerged from disasters.

Project Methods: The PubMed and Ovid databases were searched for articles documenting origination of plastic surgery ideas during a disaster with the terms "disaster", "war", "plastic", "burn", "terror", and/or "novel". Types of disasters considered include: war, terrorism, accident and natural disaster. Articles were included if they discussed novel therapies published emerging from a disaster.

Results: Twenty-one articles meeting inclusion criteria. Eleven papers described innovations during war including use of k-wires in hand fractures, gender affirmation surgery, delayed wound closure, cleft lip repair, vascular repair of acute trauma, sulfamylon antibiotics, and portable vacuum-assisted-closure (VAC) devices. Two articles resulted in surgical staff collaboration modeling and rapid recruitment from terrorist attacks. Five manuscripts defined accidents that brought about ideas of cultured epithelial autografts, VAC for burns, and targeted muscle reinnervation to decrease chronic pain in amputees. Natural disasters that caused change were depicted in three articles discussing adequate burn treatments for resource poor areas and using telemedicine for outpatient care and academic meetings.

Conclusions and Potential Impact: The field of Plastic Surgery continues to advance from innovations developed during disasters. Our study found war resulted in the greatest novel advancements. Terrorism and natural disasters have emerged as sparking innovation during the last few decades. Physicians should be encouraged to look for opportunities of innovation using their ingenuity when faced with disaster. What progress will COVID-19 bring? 